\title{
Análisis de contagio en el sistema financiero mexicano combinando el modelo de Merton y redes aleatorias
}

\author{
Analysis of contagion in Mexican financial system combining Merton \\ and random networks models \\ Guillermo Sierra Juárez \\ Universidad de Guadalajara, México
}

Recibido el 11 de diciembre de 2014; aceptado el 22 de mayo de 2015

Disponible en Internet el 17 de noviembre de 2016

\begin{abstract}
Resumen
En el contexto de la regulación y de los acuerdos de Basilea resalta la importancia de controlar el riesgo sistémico y los efectos de contagio. El presente trabajo propone un modelo que consiste de dos fases (considerando el modelo de Merton en la primera parte y un modelo Erdös-Rényi de redes aleatorias en la segunda) para analizar el contagio en el sistema bancario mexicano. Al final se concluye que los bancos pueden ser contagiados a partir de un default inicial y caer en incumplimiento o bien no verse afectados por el evento. Lo anterior dependerá de la volatilidad y del crecimiento de los pasivos y de los parámetros propios de las redes, como son la severidad de choque, el número de nodos afectados inicialmente, la estructura de la red (homogénea o heterogénea), la proporción (activos externos/activos) interbancarios y la proporción capital/activos. Los resultados muestran consistencia con la política de regulación que se lleva a cabo en México para evitar un efecto de contagio en sistema financiero.

(C) 2016 Universidad Nacional Autónoma de México, Facultad de Contaduría y Administración. Este es un artículo Open Access bajo la licencia CC BY-NC-ND (http://creativecommons.org/licenses/by-nc-nd/4.0/).
\end{abstract}

Códigos JEL: G01; G21; G33

Palabras clave: Contagio; Erdös-Rényi; Merton; Redes aleatorias

\begin{abstract}
The control of systematic risk and the contagion effect are very important on the regulation and Basilea context. This paper presents a two phase model (Merton Model on the first part and Random networks Erdös-Rényi in the second part) in order to analyze the contagion effect on the Mexican bank system. The
\end{abstract}

Correos electrónicos: gsierraj@yahoo.com.mx, gsierraj@cucea.udg.mx

La revisión por pares es responsabilidad de la Universidad Nacional Autónoma de México. 
conclusions are that the Banks could not be infected or fall into default depending of the behavior of liabilities, the structure of the network and (external asset/interbank asset) ratio and (capital/asset) ratio. The results are consistent with the regulation politics in order to avoid the contagion in Mexico Financial System.

(C) 2016 Universidad Nacional Autónoma de México, Facultad de Contaduría y Administración. This is an open access article under the CC BY-NC-ND license (http://creativecommons.org/licenses/by-nc-nd/4.0/).

JEL classification: G01; G21; G33

Keywords: Contagion; Erdös-Rényi; Merton; Random networks

\section{Introducción}

El objetivo del presente trabajo es realizar un análisis del efecto contagio en el sistema bancario mexicano utilizando una variante del modelo de Merton y la técnica conocida como redes aleatorias. La intención de esta propuesta es confirmar que la regulación bancaria y en particular los requerimientos de capital son fundamentales para evitar los contagios en el sistema financiero y el control del riesgo sistémico.

La metodología que se utiliza principalmente es la teoría financiera en el contexto de BlackScholes y la teoría de redes aleatorias. El modelo propone explicar el efecto de contagio combinado dos fases: en la primera de ellas (el choque inicial) se determina la probabilidad de incumplimiento o de quiebra de un banco utilizando el modelo de Merton-Credit-Monitor, que se utiliza como detonador o choque inicial y que determina el número inicial de nodos o bancos afectados. En la segunda fase, a partir del modelo de contagio referido en varios artículos previos, principalmente el de Dasgupta y Kaligounder (2014), se hace una simulación del contagio a los restantes bancos utilizando fundamentalmente el modelo de Erdös-Rényi, donde poco a poco y de acuerdo a una tasa propuesta se va esparciendo el contagio y se va reduciendo el capital de las instituciones afectadas provocando la quiebra o la entrada en default de algunos bancos. El modelo también requiere otros parámetros, como son la proporción de activos externos/activos interbancarios, la proporción capital/activos, el tipo de choque idiosincrático o correlacionado, el grado de los nodos y la distribución homogénea o heterogénea de la red.

El trabajo comienza con una revisión de los principales trabajos de redes aleatorias, pasando desde el modelo de Erdös-Rényi (1960) y las aportaciones fundamentales de Newman (2003) a la teoría de grafos y redes aleatorias, así como sus trabajos en epidemiología SIR (Newman, 2002). Posteriormente se hace referencia, entre otros, a los trabajos más relacionados con redes aleatorias, como los de Nier y Gai (2010), y los aplicados al caso de México, hasta llegar al artículo de Dasgupta y Kaligounder (2014).

En la parte I se coloca el problema del riesgo sistémico y del contagio en el contexto internacional y nacional de la regulación internacional, haciendo una revisión de los acuerdos de Basilea, sus principales modificaciones, la importancia de los requerimientos de capital y la situación propia del sistema financiero de México.

Una vez justificada su importancia en el contexto académico y práctico de la regulación, el trabajo muestra un revisión cronológica de las últimas décadas de la banca, desde su nacionalización, privatización, crisis financieras, el caso del Fondo Bancario de Protección al Ahorro (FOBRAPROA), la entrada de bancos extranjera, el Tratado de Libre Comercio de América del Norte (TLCAN), la llegada de bancos de procedencia extranjera y el impulso del gobierno para la desconcentración. Además de mencionar el crecimiento en el registro de varios nuevos bancos 
enfocados en un nicho distinto de la población, además de mostrar la evolución histórica de los activos pasivos y capital contable.

En la parte II se revisan los modelos a utilizar, iniciando con el de Merton y el Credit Monitor y su fundamento en la teoría de Black-Scholes y posteriormente los modelos basados en redes aleatorias Erdös-Rényi para plantear el contagio y combinando el resultado del modelo de dos fases (modelo de Merton-Credit-Monitor y Erdös-Rényi y Dasgupta y Kaligounder para contagio) y en seguida se mencionan los resultados.

Al final se presentan las conclusiones generales basadas en el trabajo propuesto, además de la bibliografía y un apéndice los bancos existentes en México en 2014.

\section{Parte I}

\section{Antecedentes}

El contagio en instituciones de crédito (particularmente bancos comerciales) es un tema que se ha analizado desde un enfoque práctico y también académico. Entre las diferentes formas de analizar el efecto contagio existe una herramienta que ha sido de mucha utilidad recientemente y que es conocida como redes aleatorias. A continuación se hace una breve revisión de artículos relacionados al tema.

En Newman (2003) se explica el modelo de grafos o redes aleatorios con bastante claridad y los modelos Erdös-Rényi (también se puede revisar el trabajo original: Erdös y Rényi, 1960), posteriormente en el mismo trabajo propone el modelo de red del mundo real y explica que la diferencia obedece a la ausencia de clústers de redes o transitividad y a una distribución no muy realística de tipo Poisson. También se describen aplicaciones a problemas de robustez de redes y aplicaciones a la dispersión de epidemias. En Newman (2002) se muestra una gran clase de modelos estándar epidemiológicos (SIR) que pueden ser resueltos para una gran cantidad de casos.

En Nier, Yang, Yorulmazer y Alentorn (2007) se revisa como el riesgo sistémico, que es el riesgo común en todo el mercado (y no puede ser diversificado), puede generar inestabilidad en toda la estructura financiera que incluso puede producir un efecto de cascada y hundir el mercado. En el diseño se propone un sistema bancario compuesto por los bancos como nodos y las líneas relacionales como las conexiones interbancarias. Se van cambiando los parámetros claves, principalmente los niveles de capitalización, el nivel de conexión de los bancos y el grado de concentración del sistema. El autor encontró que a mayor nivel de capitalización, más resistente es el sistema al efecto de contagio, el efecto de grado de conectividad; un pequeño incremento aumenta el efecto del contagio pero después del umbral la conectividad mejora la habilidad del sistema bancario de absorber el choque, el tamaño de los pasivos incrementa el riesgo de default y los sistemas bancarios más concentrados son más propensos a mayores riesgos sistémicos. Este artículo es muy semejante a la referencia básica seguida en el presente trabajo de Dasgupta y Kaligounder (2014).

En Estrada y Morales (2007) se examina la estructura de capital del mercado interbancario de Colombia y, a partir de un modelo de simulación durante el periodo 2005-2007, se analiza el comportamiento del riesgo de contagio, que se define como el riesgo que enfrenta una entidad de no poder satisfacer su demanda de liquidez en el mercado cambiario a causa de choques de liquidez en la demás entidades. Los autores encontraron un incremento en el contagio debido a una menor capacidad de absorción en las entidades en el periodo analizado. 
En Gai y Kapadia (2010) se desarrolla un modelo de contagio en redes financieras con estructura arbitraria y se explora como el potencial de impacto es influenciado por los choques idiosincráticos y agregados y por la liquidez del mercado. Se encuentra que el sistema exhibe una tendencia frágil de la robustez y estima la probabilidad de contagio.

En Martínez-Jaramillo, Kabadjova, Benitez y Solorzano (2012) se presenta un estudio empírico del sistema bancario mexicano y sus implicaciones para el riesgo sistémico. Se proponen mediciones no topológicas para describir el comportamiento y la evolución de los bancos en una red, y se crea una medida de interconexión. Al final se encuentra que la red de sistemas de pagos está conectada más fuertemente que la red de exposición bancaria.

En Klinger y Teply (2014) se modela el riesgo sistémico de los bancos con un modelo de redes y utiliza prueba de stress para diferentes medidas de riesgo. Por medio de simulaciones confirma que la suficiencia de capital en cada banco es crucial para la estabilidad del sistema, pero no es suficiente cuando el sistema ya ha entrado en colapso.

En Gleeson, Hurd, Melnik y Hackett (2013) se aplica un método de redes con una distribución de grado arbitrario y se inicializa por el default de uno o más bancos (se incluyen los efectos de riesgo liquidez). Los resultados obtenidos son validados por simulaciones Montecarlo y pueden ser de gran ayuda para entender la estabilidad de una topología de una red bancaria

Finalmente, la referencia más importante para este trabajo es la de Dasgupta y Kaligounder (2014), que hace una extensión del modelo de red financiera propuesto por Nier et al. (2007) para escenarios de mercados over the counter (mercados de mostrador) estableciendo una medida de estabilidad global. Además, descubren interesantes implicaciones de la evaluación de las medidas de estabilidad a partir de las propiedades topológicas y de los parámetros de la red.

\section{Regulación bancaria}

Como consecuencia de las crisis económico-financieras de las últimas décadas del siglo xx, los líderes de los países más industrializados han tenido una serie de reuniones periódicas en Basilea (Suiza) con la finalidad de llegar a consensos en materia de regulación financiera que sirvan, entre varios objetivos, a la prevención de grandes crisis económico-financieras mundiales, consensos que se conocen como los acuerdos de Basilea. En esta sección se hace una breve presentación de estos acuerdos en el contexto mundial y en particular de México. (Para una consulta más profunda de Basilea I, II o III se puede consultar el sitio electrónico del Comité de Basilea.)

Los acuerdos de Basilea son un conjunto de recomendaciones para establecer un capital mínimo que una entidad financiera debe tener en función de los riesgos a los que se encuentra expuesto. El grupo de los países que encabezaron estos acuerdos está integrado por los gobernadores de los bancos centrales de Alemania, Bélgica, Canadá, España, Estados Unidos, Francia, Italia, Japón, Luxemburgo, Holanda, el Reino Unido, Suecia y Suiza. Hasta el momento se han llevado a cabo tres reuniones: Basilea I (1988), Basilea II (2004) y Basilea III (2009).

En el acuerdo de Basilea I se establece que el riesgo más importante es el de crédito, y se estima agrupando las exposiciones de riesgo en 5 categorías dependiendo de la contraparte y asignando una «ponderación» diferente a cada una de ellas $(0 \%, 10 \%, 20 \%, 50 \%, 100 \%)$; la suma de los riesgos ponderados conformaba los activos de riesgo. El acuerdo establecía que el capital mínimo de la entidad bancaria debería ser el $8 \%$.

En la segunda versión de los acuerdos, Basilea II, se propuso un nuevo conjunto de recomendaciones cuyos objetivos van principalmente en el sentido de buenas prácticas bancarias y la estandarización internacional. Las recomendaciones se basan en los siguientes pilares fundamentales. 
El pilar I se refiere al cálculo de los requisitos mínimos de capital y considera la calidad crediticia de los prestatarios (utilizando ratings externos o internos), y además añade requisitos de capital por el riesgo operacional. El pilar II tiene que ver principalmente con el proceso de supervisión de la gestión de los fondos propios. Finalmente, el pilar III trata sobre la disciplina de mercado. Además se establecieron normas de transparencia y se exigió la publicación periódica de información acerca de su exposición a los diferentes riesgos. En este acuerdo se define la forma de realizar estadísticas y cálculos para el cálculo del riesgo de crédito utilizando las probabilidades de incumplimiento, la severidad y la exposición al incumplimiento.

Basilea III se dio en un marco de intercambios y retroalimentaciones por parte del Comité de Basilea con diversos bancos mundiales entre diciembre de 2009 y diciembre de 2010 y en donde el grupo de los 20 países más industrializados se comprometieron a su implementación.

Como es sabido, los acuerdos que ha establecido el Comité de Basilea (en cualquiera de sus versiones) no obligan bajo ninguna acción legal a su cumplimiento en cada una de las naciones. No obstante, es responsabilidad de los órganos de supervisión de cada país considerar dichas recomendaciones e incluirlas en su regulación.

En México, la Comisión Nacional Bancaria y de Valores (CNBV) —órgano desconcentrado de la SHCP, con autonomía técnica y facultades ejecutivas- es la que en su ámbito de competencia incluyó en la Circular única de Bancos (CUB) los términos y requerimientos de los acuerdos de Basilea con el fin de que las instituciones financieras pudieran realizar su implementación.

Hasta 2012, la CUB concentraba 354 artículos que los bancos deberán observar para la ejecución de sus operaciones; la mayor parte de estos artículos concentra aspectos de crédito.

La modificación de la regulación Basilea III (ver CNBV, 2013, Reporte de alertas tempranas) empezó su implantación a partir de 2012 y las medidas adoptadas (incluso anticipadamente) fueron: a) la mejora en la calidad de capital; b) la inclusión del suplemento de conservación; c) el aumento de ponderadores de riesgo para activos complejos, y d) la mejora en las características de las obligaciones subordinadas para absorber pérdidas. Para lograr esto, en México se realizarán las siguientes modificaciones: 1) nueva definición e integración del capital; 2) redefinición del marco de alertas tempranas, y 3) criterios para la inclusión de obligaciones subordinadas en el capital.

Para el caso de México, las medidas de Basilea III se empezaron a implementar a partir de 2012, como se mencionó anteriormente; esto fue una adopción anticipada con respecto al calendario establecido en Basilea III y ocurrió en un momento en que los índices de capitalización que tiene los bancos mexicanos se encontraban por arriba de los mínimos propuestos. La CNBV afirmó que las nuevas reglas no tendrán un impacto significativo para ningún banco del sistema; previo a la implementación de las medidas de Basilea III se tenía la perspectiva de que dicha implementación representaría una reducción mínima del índice de capitalización (ICAP) de la banca múltiple en México, y esto como consecuencia de que la reglas vigentes antes de la nueva implementación ya eran muy parecidas

\section{Historia reciente y breve descripción de la banca en México}

Pocos años después de la privatización, en 1995 el sector bancario fue uno de lo que más resintió los efectos de la crisis en México. Altos índices de morosidad provocados por altas tasas de interés, contracción de los fondos prestables, disminución en el nivel de intermediación financiera, así como la desaceleración económica hicieron necesaria la intervención del Estado en la capitalización de dichas instituciones (los datos y la información de esta sección se basan en el trabajo de Hernández, 2001). 
Ante esta situación el gobierno buscó implantar una serie de programas destinados al fortalecimiento del sector bancario a través del programa conocido como Fondo Bancario de Protección al Ahorro (FOBAPROA). Inicialmente se creó un fondo de capitalización temporal con la finalidad de dar respuesta rápida a los problemas de insolvencia y quiebra de las instituciones financieras. Posteriormente, debido al grave deterioro de la cobranza en los bancos, el gobierno llevó a cabo un programa de compra de cartera en el cual se comprometió a adquirir 2 pesos de cartera vencida por cada peso adicional que los accionistas aportaran a la capitalización del banco; estos créditos fueron adquiridos por medio de pagarés del FOBAPROA con el aval del gobierno federal. Los bancos mantuvieron la responsabilidad de cobrar los créditos que vendieron del FOBAPROA, obligándose a compartir las pérdidas provenientes de cartera incobrable.

En lo referente a la regulación, se obligó a los bancos a elevar su nivel de provisiones sobre la cartera vencida, y como consecuencia del Tratado de Libre Comercio de América del Norte (TLCAN) en 1995 se modificaron las restricciones respecto a la participación del capital extranjero con la finalidad de aumentar la capitalización del sector. Este capital ha venido desempeñando un importante papel en la recapitalización de los bancos que permitió reducir la magnitud de la crisis y disminuir el costo fiscal del rescate. En 1998 se aprobó convertir los pasivos del FOBAPROA en deuda pública, y posteriormente serían transferidos al Instituto de Protección al Ahorro Bancario (IPAB) los activos que fueran producto de operaciones válidas. El IPAB es desde entonces el encargado de supervisar la recuperación de los activos y de la cartera vencida de los bancos que fueron respaldados por el FOBAPROA, y además es el encargado de administrar los seguros de depósito.

Después de la privatización, la mayoría de las instituciones de banca múltiple sufrieron fuertes cambios: unas cambiando varias veces de dueño, otras siendo intervenidas por el gobierno y otras tantas desapareciendo. Por ejemplo, en 1999 solo el 35\% de los 20 bancos que operaban provenían de las privatizaciones que se había realizado en los años previos. Posteriormente, la participación de extranjeros en la banca en la captación creció de forma importante, en un inicio con la incorporación del Banco Bilbao Vizcaya (en 2000 BBVA capitaliza a Bancomer), Banco Santander (adquiere en 1997 Iverméxico y en 2000 logra la adjudicación de Banco Serfin) y City Bank (en 2001 el Grupo Financiero Banamex es comprado por City Group).

De acuerdo a datos de la CNBV, hasta el 2003 operaban en México 19 bancos extranjeros, los cuales controlaban el $81.6 \%$ de los activos totales del sistema bancario; de estos bancos, BBVA/Bancomer y Banamex/Citibank controlaban la gran mayoría del mercado.

La creación de nuevos bancos y la participación de capital externo han provocado que el nivel de concentración, ya sea medido por los activos y/o por la captación, fuera disminuyendo gradualmente.

Dando continuidad a los procesos de desregulación, apertura, incremento de la competencia y segmentación del mercado bancario, entre los años 2002 y 2008 la Secretaria de Hacienda y Crédito Público (SHCP) autorizó la creación de 16 nuevos bancos. La mayoría de estas instituciones bancarias (15) son consideradas pequeñas debido al monto de sus activos: el más grande de esos bancos es Banco Azteca, el cual cuenta con un activo que apenas representa el $7.5 \%$ de BBVA/Bancomer, que era el banco múltiple más grande en México.

De acuerdo a Solorza (2008), a estos nuevos bancos también se les denomina «bancos de nicho», por estar enfocados a la banca de menudeo y porque sus operaciones se limitan a espacios geográficos locales o regionales —excepto Bancoppel y Banco Azteca, que cuentan con una red de sucursales nacional ligada a sus tiendas Coppel y Elektra; incluso el segundo opera en otros países de América Latina- y a segmentos o productos específicos que en algunos les permiten sobrevivir y en otros crecer. 


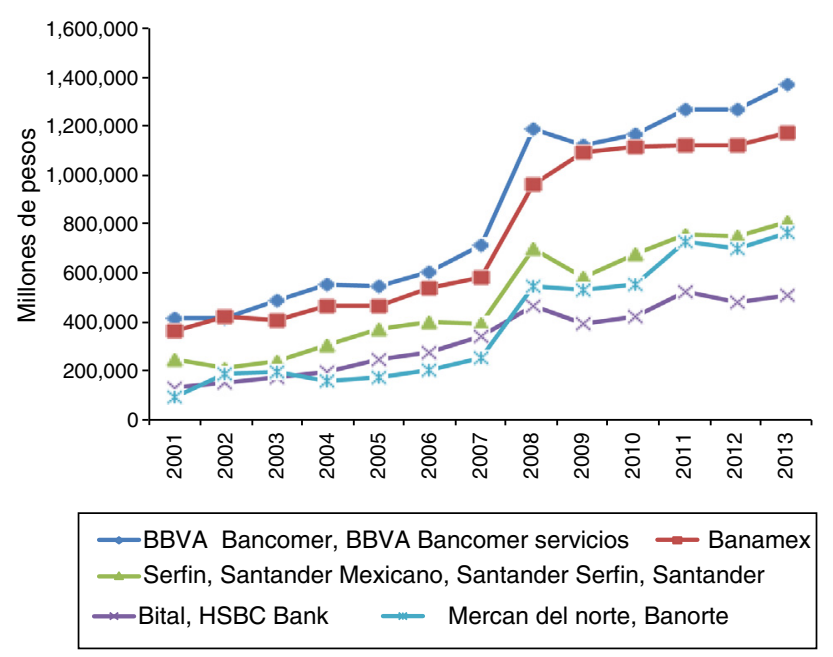

Figura 1. Principales bancos en México (activos).

Fuente: elaboración propia a partir del portafolio de información de la SHCP.

Sin embargo, la mayoría de estos bancos están ligados a grupos empresariales cuyas actividades económicas muestran que pretenden bancarizar sectores poblacionales que considerando únicamente los ingresos de algunos de los miembros de la familia no califican para obtener créditos en los grandes bancos múltiples, pero sumados ya como ingresos familiares sí reúnen los requisitos. Así, Banco Azteca, Bancoppel, Ahorro Famsa, Fácil y Wal-Mart están posesionados en el sector comercial de electrodomésticos, ropa y comestibles; Banco Amigo forma parte de un grupo de empresas (Grupo Landus, desarrollador inmobiliario) dedicadas al desarrollo de comunidades donde se conjuntan áreas habitacionales, hoteleras, comerciales, industriales y de servicios. Algunos de estos bancos están ligados a grupos financieros y se ocupan del mercado corporativo, como son los casos de Banco Multaba, Banco Prudential y Banco Regional (como lo han venido haciendo los bancos creados en los años noventa). Banco Compartamos es una institución que proporciona crédito a microempresas conformadas por mujeres pobres. Banco Volkswagen claramente se dirige al financiamiento automotriz, y solamente Banco Autofiń mantiene una estrategia de negocios combinada. La mayoría de los nuevos bancos son considerados «bancos de nicho» por especializarse en algunos productos y segmentos de mercado y porque en sus inicios se dedicaron a otorgar crédito a sus clientes para aumentar sus ventas de electrodomésticos. Sin embargo, como el sector poblacional al que se dirigen, estas instituciones bancarias se encuentran ubicadas socioeconómicamente en los estratos medio y bajo.

A continuación se presenta de forma gráfica la evolución financiera de los principales bancos de México en el periodo 2001-2013 y con información obtenida de los boletines estadísticos de la CNBV. Se debe considerar que se consolidan en este comportamiento histórico en su respectivo momento: 1) BBVA Bancomer con BBVA Bancomer Servicios; 2) Serfin, Santander Mexicano, Santander Serfin y Santander; 3) Bital con HSBC y, más recientemente, 4) Banorte con Ixe. En las figuras 1-3 se presenta el crecimiento de los activos, pasivos y capital contable de los principales bancos en México: BBVA Bancomer, Banamex, Santander, HSBC y Banorte. Se aprecia claramente el dominio y el crecimiento de los dos principales bancos en México, BBVA Bancomer, Banamex, Santander y Banorte. 


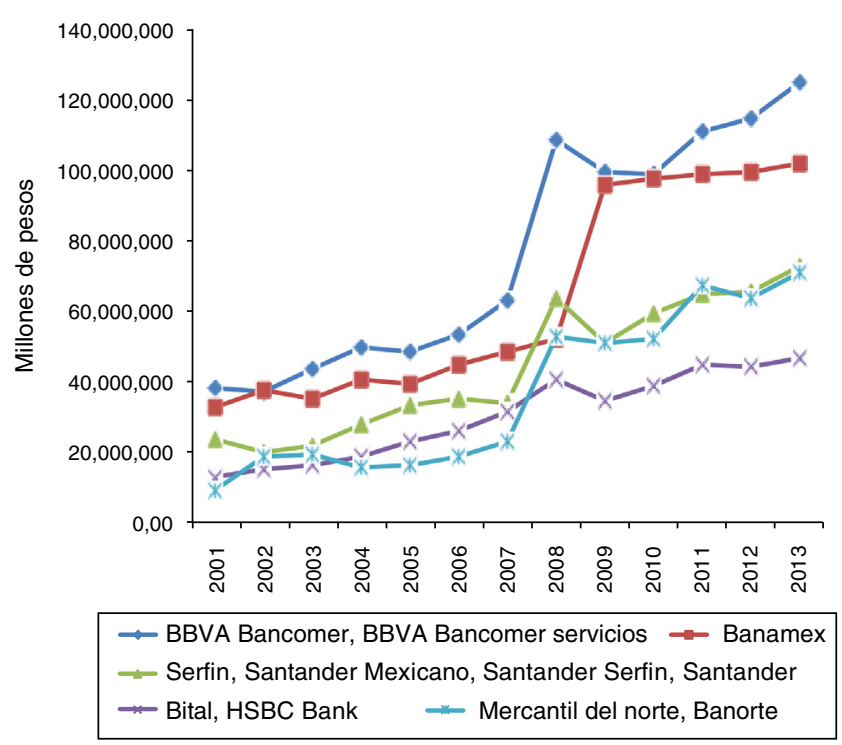

Figura 2. Principales bancos en México (pasivos).

Fuente: elaboración propia a partir del portafolio de información de la SHCP.

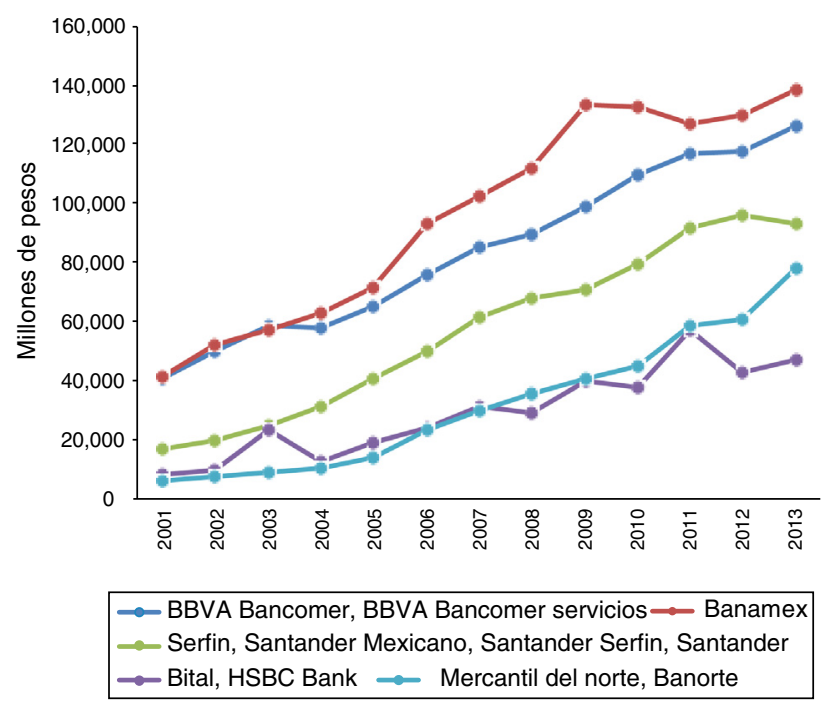

Figura 3. Principales bancos en México (capital).

Fuente: elaboración propia a partir del portafolio de información de la SHCP.

\section{Parte II. El modelo}

Dentro del estudio del riesgo de crédito existen varios modelos para determinar las probabilidades de incumplimiento que van desde los spreads o sobre tasas, pasando por los modelos no condicionales como el paramétrico, el Credit metrics, el Credit Risk+ y los modelos condicionales, como el Credit Monitor, el modelo de Merton y el Credit Porfolio View. Otro modelo 
de características muy prácticas es el modelo de la Z-score de Altman, que emplea las razones financieras ponderadas de acuerdo a una regresión lineal de información histórica de empresas que han caído en incumplimiento. Al final se determina una Z-score que determinará si la empresa se encuentra en zona segura, zona gris o zona de posible quiebra.

De todos los modelos que existen, el que se emplea en este trabajo, de acuerdo a las características e información disponible, es el modelo de Merton con un pequeño ajuste en las variables conocidas, y que también es conocido como Credit-Monitor.

\section{Modelo de Merton}

El modelo de Merton se clasifica como de tipo estructural y supone que los inversionistas tienen información perfecta del mercado, conocimiento completo del valor de los activos y de la deuda de todas las compañías. El incumplimiento se presenta cuando el valor de los activos es inferior al monto total de la deuda financiera. Dicho valor de los activos se considera como el valor de una opción call europea que tiene como precio de ejercicio el monto de la deuda, y se utiliza la fórmula de valuación de Black y Scholes para calcular la probabilidad de incumplimiento. El modelo de Merton supone que una empresa emite bonos cupón cero que vencen en una fecha establecida T. (La descripción del modelo está basada en Sierra y García, 2013.)

Una variante del modelo de Merton fue desarrollada por KMV y se le conoce como Credit Monitor; en principio la idea es la misma, pero ahora las variables corresponden a las de un balance financiero en un cierto momento del tiempo t y se satisface en todo momento la expresión (ver también Sierra y García, 2013):

$$
A_{t}=C_{t}+P_{t}
$$

donde $A_{t}$ : activo total de la empresa en tiempo $t ; C_{t}$ : capital de la empresa en tiempo $t ; P_{t}$ : pasivo de la empresa en tiempo $t$ (principalmente el valor de la deuda en el tiempo $t$ ).

En este caso se plantea de la misma manera que en las opciones financieras que contaremos con un monto de capital si al final del periodo $t$ los activos son mayores que los pasivos; en caso contrario, se contará con cero de capital:

$$
\mathrm{C}_{\mathrm{T}}=\max \left(\mathrm{A}_{\mathrm{T}}-\mathrm{P}_{\mathrm{T}}, 0\right)
$$

Siguiendo la formulación conocida de Black-Scholes con un cambio de variable se llega a:

$$
\mathrm{C}_{\mathrm{t}}=\mathrm{A}_{\mathrm{t}} \Phi\left(\mathrm{d}_{1}\right)-\mathrm{P}_{\mathrm{T}} \mathrm{e}^{-\mathrm{r}(\mathrm{T}-\mathrm{t})} \Phi\left(\mathrm{d}_{2}\right)
$$

donde

$$
\begin{aligned}
& \mathrm{d}_{1}=\frac{\ln \left(\frac{\mathrm{A}_{\mathrm{t}}}{\mathrm{P}}\right)+\left(\mathrm{r}+\frac{1}{2} \sigma_{\mathrm{v}}^{2}\right)(\mathrm{T}-\mathrm{t})}{\sigma_{\mathrm{V}} \sqrt{\mathrm{T}-\mathrm{t}}} \\
& d_{2}=d_{1}-\sigma_{v} \sqrt{T-t}
\end{aligned}
$$

Y para obtener la probabilidad de incumplimiento del pago de la deuda, se tiene:

$$
\mathbb{P}\left\{\mathrm{A}_{\mathrm{T}}<\mathrm{P} \mid \mathrm{A}_{\mathrm{t}}\right\}=1-\Phi\left(\mathrm{d}_{2}\right)=\Phi\left(-\mathrm{d}_{2}\right)=\int_{-\infty}^{\mathrm{d}_{2}} \frac{1}{\sqrt{2 \pi}} \mathrm{e}^{-\mathrm{x}^{2} / 2} \mathrm{dx}
$$


La razón principal por la cual se realiza el ajuste de las variables sobre las razones financieras como activo, pasivo y capital, es que esta información está al alcance público.

De lo anterior, si conocemos los activos en el tiempo $t$ de un banco, si tenemos una proyección de sus pasivos y se conoce la volatilidad de los activos y la tasa de interés, entonces podríamos estimar la probabilidad de incumplimiento de dicho banco como una descompensación del crecimiento de sus pasivos.

\section{Modelos de redes aleatorias}

En su trabajo seminal Paul Erdös y Alfred Rényi (Erdös y Rényi, 1960) propusieron uno de los primeros modelos teóricos de redes, los grafos aleatorios. Este sencillo modelo consiste de nodos o vértices unidos por líneas de enlace o links entre cada pareja de vértices elegidos de forma aleatoria. El modelo más comúnmente estudiado es el denotado como $\mathrm{G}_{\mathrm{n}, \mathrm{p}}$, en el cual cada posible línea que une dos vértices tiene una probabilidad independiente $p$ de estar conectado y de no estar conectado de $1-p$. Frecuentemente se desea expresar $\mathrm{G}_{\mathrm{n}, \mathrm{p}}$ no en términos de $p$, sino del grado promedio de un vértice, $z$, el grado de un vértice que es el número de líneas conectadas al mismo vértice. El grado promedio de un vértice que tiene líneas finales está dado por (esta sección se basa en Newman, 2003):

$$
z=\frac{n(n-1) p}{n}=(n-1) p \approx n p
$$

Entonces cualquier propiedad expresada en términos de $p$ también puede expresarse en términos de $z$.

\section{Modelo Erdös-Rényi}

Los grafos aleatorios de Erdös-Rényi tienen varias propiedades deseables para el modelado de redes. Por ejemplo, en su trabajo original encontraron que el modelo propuesto tenía propiedades de transición de fase, es decir, conforme $z$ se incrementa se puede producir una forma conocida como componente gigante. Para pequeños valores de $z$ hay pocas líneas de conexión en la gráfica y, por tanto, hay muchos nodos desconectados. Las componentes pequeñas en general son constantes, pero hay un valor en $z$ a partir del cual componentes mayores en la gráfica contienen una fracción muy grande del número total de vértices, es decir, el tamaño se crece linealmente con el tamaño total de la gráfica. Este tipo de componentes se conoce como componente gigante, y su formación es una evocación del comportamiento de las gráficas del mundo real.

Sin embargo, dos diferencias han sido detectadas en la literatura (Watts y Strogatz, 1998) ${ }^{1}$. La primera, en las redes del mundo real se muestra un fuerte clustering ${ }^{2}$ o transitividad que no se presenta en los modelos Erdös-Rényi. La segunda diferencia es en sus distribuciones de grado. La probabilidad $p_{k}$ de que un nodo en una gráfica aleatoria Erdös- Rényi tenga exactamente un grado

\footnotetext{
1 Ellos establecieron un llamado coeficiente de clustering.

2 Se conoce como «clustering» si la probabilidad de dos vértices de estar conectados por una línea es mayor cuando los vértices en cuestión tienen un vecino común.
} 
$k$ está dada por la distribución binomial que en el límite $n \gg k z$, se convierte en una distribución Poisson.

$$
\begin{aligned}
& p_{k}=\left(\begin{array}{c}
n-1 \\
k
\end{array}\right) p^{k}(1-p)^{n-1-k} \\
& p_{k}=\frac{z^{k} e^{-z}}{k !}
\end{aligned}
$$

Ambas distribuciones (binomial y Poisson) tienen características similares, ya que poseen un pico muy marcado en su distribución alrededor de su media y tienen una larga cola que decae como $1 / k$ ! Las distribuciones empíricas del mundo real son muy distintas a las anteriores, no presentan el rápido decaimiento de las distribuciones de Poisson, y esto se refleja y tiene profundos efectos en la red.

El uso de funciones generadoras de probabilidad es muy útil para deducir propiedades de las gráficas aleatorias (Newman, 2003). Supongamos una distribución de probabilidad $p_{k}$, la cual es la distribución de grados de vértices en una gráfica. La función generadora correspondiente estaría dada por:

$$
G_{0}(x)=\sum_{k=0}^{\infty} p_{k} x^{k}
$$

Esta función captura toda la información de la distribución original $p_{k}$ :

$$
p_{k}=\left.\frac{1}{k !} \frac{d^{k} G_{0}}{d x^{k}}\right|_{x=0}
$$

Para el caso de una gráfica aleatoria Erdös-Rényi con distribución de grado Poisson tenemos que $\mathrm{G}_{0}$ :

$$
G_{0}(x)=e^{-z} \sum_{k=0}^{\infty} \frac{z^{k}}{k !} x^{k}=e^{-z(z-1)}
$$

La función generadora $G_{1}(x)$ para vértices alcanzados por una línea también puede ser alcanzada:

$$
G_{1}(x)=\frac{G_{0(x)}}{z}=e^{-z(z-1)}
$$

Se puede observar que las gráficas Erdös-Rényi son muy fáciles de resolver analíticamente y tienen la propiedad de una distribución Poisson $G_{0}(x)=G_{1}(x)$.

Existe un tipo particular de gráficas en donde las líneas de enlace presentan una dirección. Este tipo de redes son más complejas que las de su contraparte de líneas no dirigidas, ya que cada vértice en una red dirigida tiene dos grados: los grados de entrada, que son el número de líneas que llegan, y los grados de salida, que son el número de líneas relacionales o links que salen del nodo. Por lo tanto, ahora se tienen dos grados de distribución, por lo que debemos esperar una distribución conjunta $p_{j k}$ que correspondería a la probabilidad de que un vértice elegido aleatoriamente tenga $j$ grados de entrada y $k$ grados de salida.

Por otra parte, un vértice A cualquiera puede pertenecer a 4 tipos de componentes: a) las componentes de entrada que es el conjunto de vértices desde el cual A puede ser alcanzada; 
b) las componentes de salida, que es el conjunto de vértices que pueden ser alcanzados desde $\mathrm{A}$; c) los componentes conectados fuertemente, que es el conjunto de vértices desde los cuales A puede ser alcanzado y en los cuales puede ser alcanzados desde A, y d) los componentes conectados débilmente, que es el conjunto de vértices que pueden ser alcanzados desde A ignorando la naturaleza de la dirección de las líneas adjuntas.

La correspondiente función generadora para una gráfica con dirección y una distribución de grado $p_{j k}$ conjunta es:

$$
\mathfrak{H}(x, y)=\sum_{j, k=0}^{\infty} p_{j k} x^{j} y^{k}
$$

La función cumple con la condición de normalización, es decir, $\mathfrak{H}(1,1)=1$, y las medias de las distribuciones de entrada y de salida están dadas por las derivadas respecto de $\mathrm{x}$, y. Sin embargo, hay solo un grado medio de $z$ para la gráfica dirigida, lo que significa que el número promedio de entradas y salidas de líneas es el mismo. Entonces se tiene una restricción en la función generadora:

$$
\left.\frac{\partial \mathfrak{H}}{\partial x}\right|_{x . y=1}=z=\left.\frac{\partial \mathfrak{H}}{\partial y}\right|_{x . y=1}
$$

y una restricción sobre la distribución de probabilidad $\mathrm{p}_{\mathrm{jk}}$ :

$$
\sum_{j, k}(j-k) p_{j k}=0
$$

Puede ahora definirse $G_{0}$ y $G_{1}$ como el número de líneas salientes de un vértice seleccionado aleatoriamente y $F_{0}$ y $F_{1}$ como el número de líneas llegando al vértice. Las funciones están dadas por:

$$
\begin{aligned}
& F_{0}=\mathfrak{H}(x .1), F_{1}(x)=\left.\frac{1}{z} \frac{\partial \mathfrak{H}}{\partial y}\right|_{y=1} \\
& G_{0}=\mathfrak{H}(1, y), G_{1}(x)=\left.\frac{1}{z} \frac{\partial \mathfrak{H}}{\partial x}\right|_{x=1}
\end{aligned}
$$

\section{Propuesta de modelo de contagio financiero}

A continuación se presenta el modelo que será utilizado en la segunda fase o fase de contagio en el sistema bancario. Algunos autores ya habían propuesto modelos semejantes (Nier et al., 2007; Gai y Kapadia, 2010; Gleeson et al., 2013), pero en esta sección se seguirá a principalmente a Dasgupta y Kaligounder (2014).

Supongamos una red bancaria representada por una gráfica o grafo dirigido con nodos y líneas de enlace ponderados $G=\{\mathrm{V}, \mathrm{E}, \Gamma\}$, donde

$\mathrm{V}$ es el conjunto de $\mathrm{n}$ bancos.

E representa el conjunto de $m$ exposiciones interbancarias dirigidas. $\mathrm{w}(\mathrm{e})=\mathrm{w}(\mathrm{u}, \mathrm{v})>0$ es la ponderación de la línea de enlace dirigida $\mathrm{e}=(\mathrm{u}, \mathrm{v})$. 
$\Gamma:\{E, I, \Upsilon\}$ son los parámetros donde E son los activos totales externos, I la exposición total interbancaria (externa e interna), A son los activos totales (E+I) y $\Upsilon$ es la proporción (capital/activos).

El modelo propone dos tipos de redes bancarias: homogéneas y heterogéneas ${ }^{3}$. Las primeras distribuyen los activos en la misma proporción entre los nodos, y en los segundos no necesariamente se distribuyen en la misma proporción. El modelo supone que los activos totales están compuestos por los activos externos y los activos interbancarios, y los pasivos están compuestos de acuerdo al modelo por los préstamos interbancarios; el valor neto capital conforma la tercera componente del balance.

La insolvencia inicial vía un choque en una red bancaria en el tiempo inicial $(t=0)$ es causada por un choque recibido a un subconjunto de nodos. El choque inicial puede ocurrir por distintos tipos de riesgo y su efecto es reducir los activos externos de un subconjunto seleccionado de bancos ${ }^{4}$. El efecto inicial del choque disminuye simultáneamente los activos externos de cada nodo por una cantidad $\mathrm{s}_{\mathrm{V}}=\Phi \mathrm{e}_{\mathrm{v}}$; por lo tanto, la reducción del valor neto $\mathrm{v}$ de su valor original $\mathrm{c}_{\mathrm{v}}$ a $\mathrm{c}_{\mathrm{v}}-\mathrm{S}_{\mathrm{v}}$, donde $\Phi>\gamma$ es el parámetro que denota la severidad del choque inicial. En adelante se referirá como mecanismo de choque a la regla de seleccionar un subconjunto inicial de nodos que recibirán dicho choque.

Denotemos por $\operatorname{deg}^{\text {in }}(\mathrm{v})$ el in-degree de nodo v; las insolvencias se propagan en unidades de tiempo discreto $t=0,1,2$. Un banco será insolvente si su valor neto modificado llega a ser negativo, y tal banco es removido de la red en el paso siguiente. Sea $V_{T}$ un conjunto de nodos que llegarán a ser insolventes antes de t cuando el choque inicial es iniciado a los nodos en $\mathrm{V}_{\mathrm{S}}$. Las insolvencias de los bancos en el tiempo $t$ afectan el capital de otros bancos en la red en el siguiente tiempo $t+1$. La propagación de insolvencia continúa hasta que un nuevo banco llega a ser insolvente.

Consideremos un modelo de red bancaria donde $\mathrm{K}$ denota la fracción de nodos en $\mathrm{V}$ que recibe el choque inicial en $\mathrm{V}$ bajo un mecanismo de choque $\Upsilon$, y sea $\xi$ sobre el promedio de nodos de la red que llega a ser insolvente para un valor dado de $\Phi \gamma$ dado un subconjunto de nodos inicial. Por ejemplo, $\xi(0.1, \mathrm{G}, 0.3,0.5$, random $)=0.9$ significa una probabilidad de que el $90 \%$ de los nodos de la red G llegará a ser insolvente con $\gamma=0.3$ y $\Phi=0.5$ si proveemos un choque a un subconjunto aleatorio del $10 \%$ de nodos de $\mathrm{G}$.

Resumiendo lo anterior, para describir una red se necesita determinar:

Tipo de red: homogéneos o heterogéneos $(\alpha, \beta)$.

Topología de la red: libre de escala directa o Erdös-Rényi.

Mecanismo del choque: idiosincrático o coordinado.

Número de nodos: entero positive.

E/I: fracción entre 0.25 y 3.5 .

$\Phi$ : fracción entre 0.5 y 0.9 .

$\mathrm{K}$ : fracción entre 0.5 y 0.9 .

$\gamma$ : entre 0.05 y $\Phi-0.05$.

Para el mecanismo del choque idiosincrático se selecciona un subconjunto de nodos distribuido uniformemente de forma aleatoria. Por otra parte, en el mecanismo de choque coordinado este

\footnotetext{
${ }^{3}$ Existen dos posibilidades 1) distribuir el $95 \%$ de los activos entre el $10 \%$ de los nodos, o 2) distribuir el $60 \%$ de los activos entre el $40 \%$ de los nodos.

${ }^{4}$ Más adelante se especifica que el choque externo pueden modelarse con Merton.
} 
mecanismo cae dentro de la categoría general de choque correlacionado donde los nodos i con un alto grado están correlacionados.

\section{Parte III}

\section{Resultados}

A continuación se presentan los resultados más importantes de la aplicación de los modelos de la sección anterior al caso del sistema bancario en México, con base en la información histórica (2001-2013) de los activos, pasivos y capital contable de los principales bancos, así como a la información más reciente (enero 2014) de los 45 bancos que hay en México (CNBV, 2014).

Se plantea un análisis de contagio modelo de redes aleatorias que consiste de dos fases. En la primera, con base al modelo Merton-Credit-Monitor, se determina la probabilidad de incumplimiento, de quiebra o de default de un banco, que depende fundamentalmente de la relación y crecimiento de sus activos y pasivos. Una vez determinado el choque inicial que da inicio a unos bancos representados por nodos afectados, se pasa a la dispersión de efecto en el sistema.

En la segunda fase, a partir del modelo y de un software basado en el contagio del sistema bancario utilizando redes aleatorias ${ }^{5}$ (Dasgupta y Kaligounder, 2014), se toma la información de la primera fase como la severidad del choque, el porcentaje de nodos afectados inicialmente, además de la información propia del tipo de red, como el número de bancos, el mecanismo de choque, la proporción (activos externos/activos interbancarios), la proporción (capital/activos), se realiza una serie de simulaciones obteniendo información sobre el contagio en todos los bancos y al final de cada proceso se obtienen los bancos que llegan al incumplimiento producto del contagio. A continuación se muestra el resumen de los resultados.

A partir de la teoría de la sección anterior y con la información de enero de 2014 (CNBV, 2014) puede determinarse la probabilidad de incumplimiento para el periodo de un año para cada uno de los 45 bancos que conforman el sistema financiero nacional. Considerando una tasa de interés libre de riesgo de 0.04 (promedio del año 2013; Banxico 2013) y considerando la volatilidad histórica de los pasivos se puede estimar, de acuerdo al modelo de Merton-Credit-Monitor, la probabilidad de incumplimiento. Al final, por razones prácticas podemos promediar el resultado de la tasa de incumplimiento de cada banco y obtener una tasa representativa con el objeto de realizar variaciones en los parámetros como la volatilidad o bien de crecimiento de los pasivos y medir el cambio en dicha probabilidad. Los resultados se muestran en las figuras 4 y 5.

En las figuras 4 y 5 se observa un incremento en la probabilidad de incumplimiento (promedio) de forma prácticamente lineal al incrementarse la volatilidad, y de manera análoga, si el porcentaje de incremento de los pasivos crece (mientras los activos permanecen igual), la probabilidad de incumplimiento también aumenta. Las tablas 1 y 2 muestran una relación de la volatilidad, el incremento de pasivos y la probabilidad de incumplimiento, o bien nodos afectados inicialmente.

Una vez estimados el número de nodos iniciales afectados por un choque de volatilidad o los incrementos inesperados de sus deudas (tablas 1 y 2) pasamos a analizar el efecto de contagio en la red de los 45 básicos.

\footnotetext{
${ }^{5}$ El software utilizado es lbre, fin_stab.jar, y fue desarrollado por Bhaskar DasGupta y Lakshmi Kaligounder en 2013(es software libre).
} 


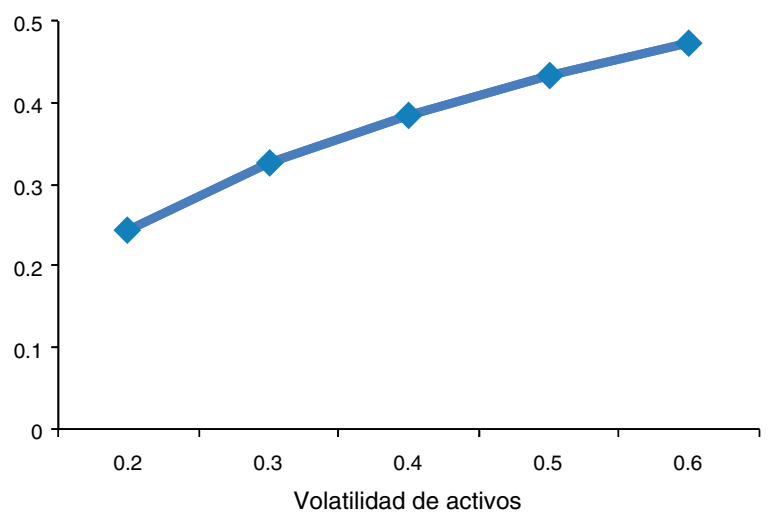

Figura 4. Probabilidad de incumplimiento como función de la volatilidad de activos. Fuente: elaboración propia.

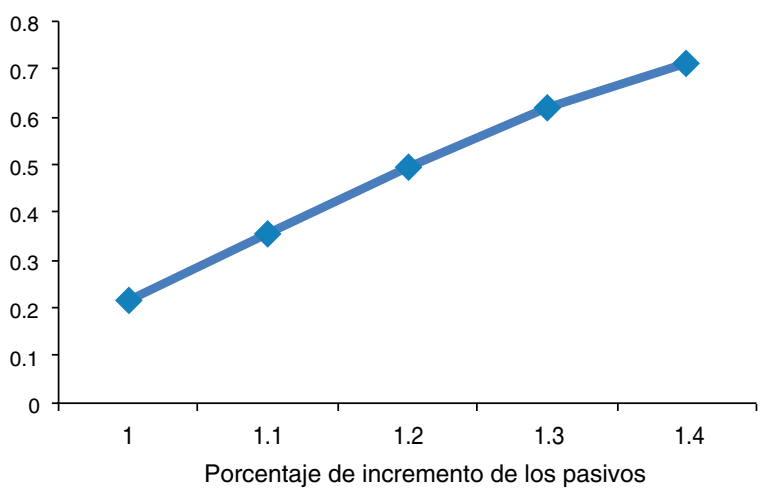

Figura 5. Probabilidad de incumplimiento como función del incremento de pasivos.

Fuente: elaboración propia.

Tabla 1

Probabilidad de incumplimiento y nodos afectados

\begin{tabular}{lll}
\hline Volatilidad (con pasivos 1) & Probabilidad de incumplimiento & $\begin{array}{l}\text { Nodos afectados inicialmente } \\
\text { (como proporción de los 45) }\end{array}$ \\
\hline 0.20 & 0.24 & 11 \\
0.30 & 0.32 & 14 \\
0.40 & 0.39 & 18 \\
0.50 & 0.43 & 20 \\
0.60 & 0.47 & 21 \\
\hline
\end{tabular}

Fuente: elaboración propia, obtenida del simulador.

\section{Modelo de contagio}

En esta fase se utiliza el modelo de Dasgupta y Kaligounder (2014). La red inicial consiste de una red aleatoria Erdös-Rényi con 45 nodos correspondientes a los bancos registrados en México. 
Tabla 2

Probabilidad de incumplimiento y nodos afectados

\begin{tabular}{lll}
\hline $\begin{array}{l}\text { Incremento en el pasivo } \\
\text { (con volatilidad de 0.20) }\end{array}$ & Probabilidad de incumplimiento & $\begin{array}{l}\text { Nodos afectados inicialmente } \\
\text { (como proporción de los 45) }\end{array}$ \\
\hline 1 & & 10 \\
1.1 & 0.22 & 16 \\
1.2 & 0.36 & 23 \\
1.3 & 0.50 & 28 \\
1.4 & 0.62 & 32
\end{tabular}

Fuente: elaboración propia, obtenida del simulador.

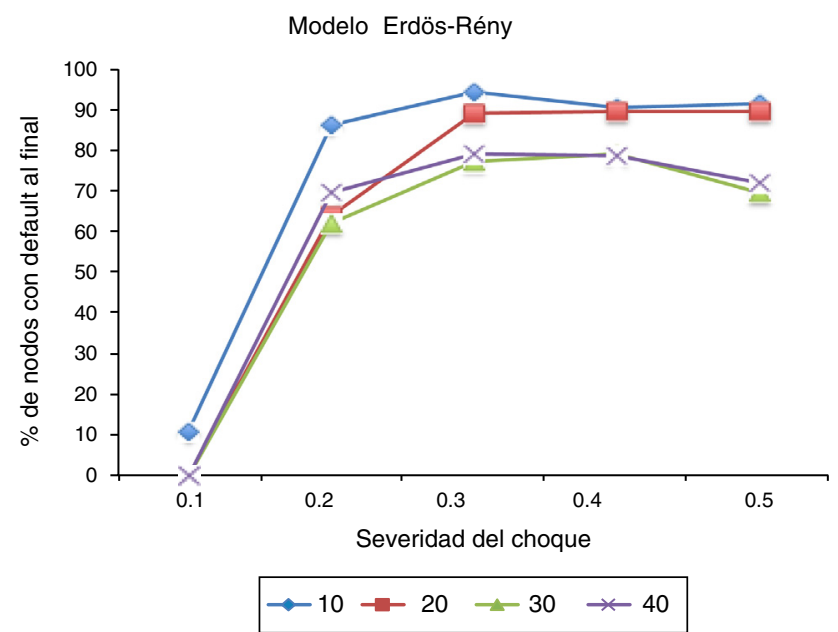

Figura 6. Número de nodos en default vs severidad de choque (choque idiosincrático, capital/activo $=0.10$ ). Modelo Erdös-Rényi.

Fuente: elaboración propia con resultado de simulador.

Se utilizara un grado de $3^{6}$ y una distribución heterogénea, es decir, que el $95 \%$ de los activos está repartido entre el $10 \%$ de los nodos ${ }^{7}$. Además, se consideran dos tipos de choques: los idiosincráticos y los correlacionados, y la proporción de activos externos interbancarios permanecerá fija en el $25 \%$. Los parámetros de severidad del choque y el número inicial de nodos afectados por el choque inicial van cambiando, y además se revisarán dos casos de la proporción capital/activos, cuando es 0.08 y cuando sea 0.10 . Posteriormente se realizan las simulaciones un número suficiente de veces ${ }^{8}$ y se obtiene al final del proceso el número promedio de bancos que van a default por ser contagiados o bien, de forma complementaria, que sobrevivieron o no fueron alcanzados por el contagio. Las figuras 6-9 muestran los resultados.

En las figuras 6-9 se observa que sería recomendable aumentar el número de simulaciones al proceso para tener mayor contundencia y robustez en los resultados, pero es una consecuencia de la limitación del programa. Al realizar el análisis individual, en el caso de los choques idiosincráticos,

\footnotetext{
6 Valor medio de conexiones.

7 Es la mejor aproximación al caso de México.

8 Debido a que en cada simulación es necesario correr el programa con todas las características propias del modelo, solo se repitió un centenar de veces.
} 


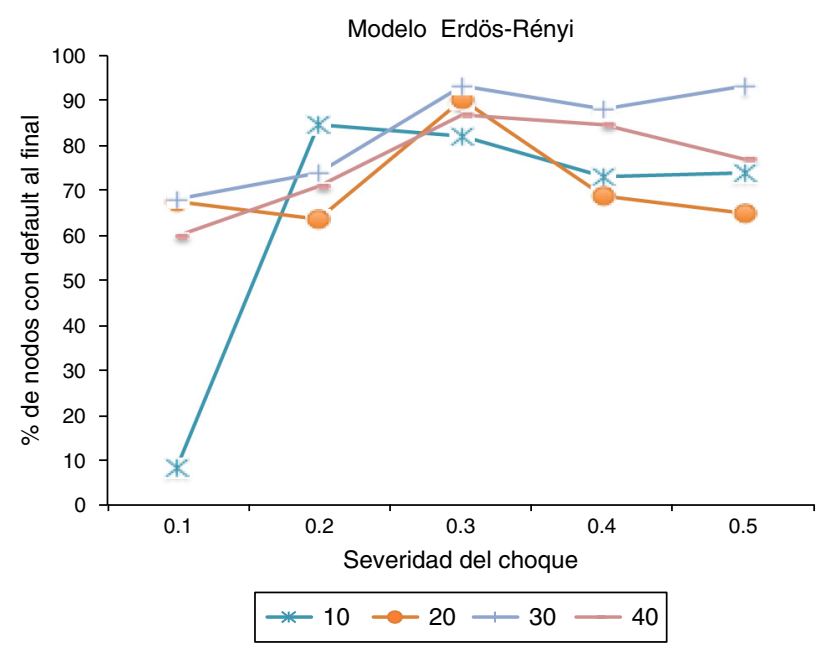

Figura 7. Número de nodos en default vs severidad de choque (choque idiosincrático, capital/activo =0.08). Modelo Erdös-Rényi.

Fuente: elaboración propia con resultado de simulador.

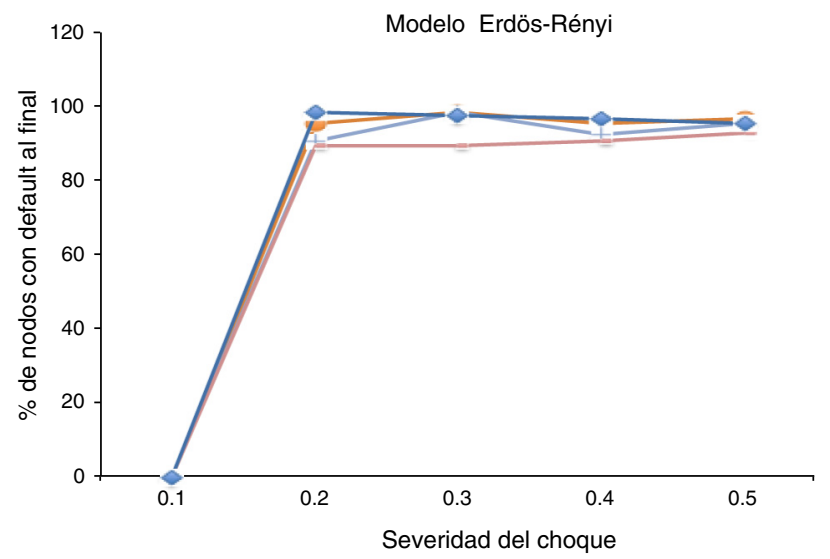

$$
\text { * } 10 \multimap 20 \div 30 \longleftarrow 40 \multimap 10
$$

Figura 8. Número de nodos en default vs severidad de choque (choque correlacionado, capital/activo =0.10). Modelo Erdös-Rényi.

Fuente: elaboración propia con resultado de simulador.

el número de nodos inicialmente afectado no provoca mucha diferencia en el número final de nodos contagiados; tampoco el grado de severidad hace variar mucho el resultado, sobre todo para severidades por encima del $20 \%$, por lo que al final todo el sistema financiero resultaría contagiado.

De los choques idiosincráticos puede decirse que si aumenta el número de nodos con default, al final del proceso, al aumentar la severidad, el comportamiento no está bien definido. Al aumentar la proporción capital/activos los resultados se vuelven más independientes de los nodos iniciales y de la severidad, y se estabiliza el comportamiento. 


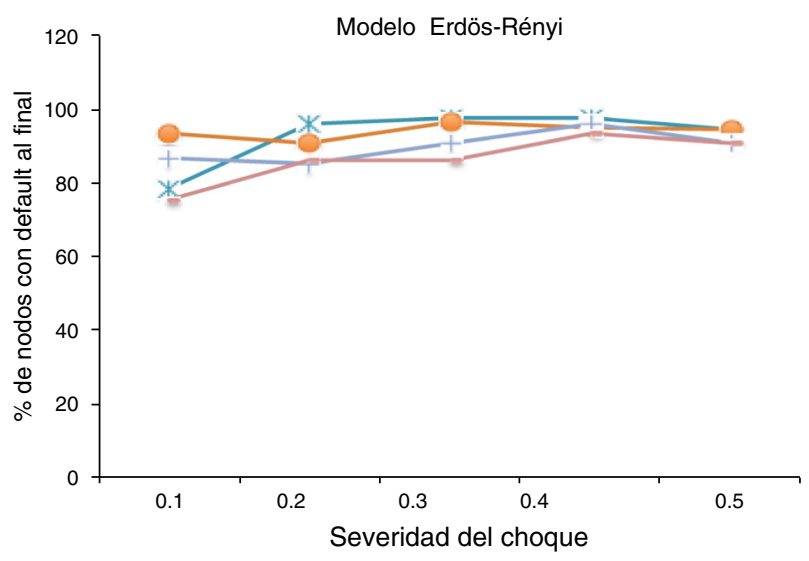

$\because-10 \multimap 20 \multimap 30 \longleftarrow 40$

Figura 9. Número de nodos en default vs severidad del choque (choque correlacionado, capital/activo =0.08). Modelo Erdös-Rényi.

Fuente: elaboración propia con resultado de simulador.

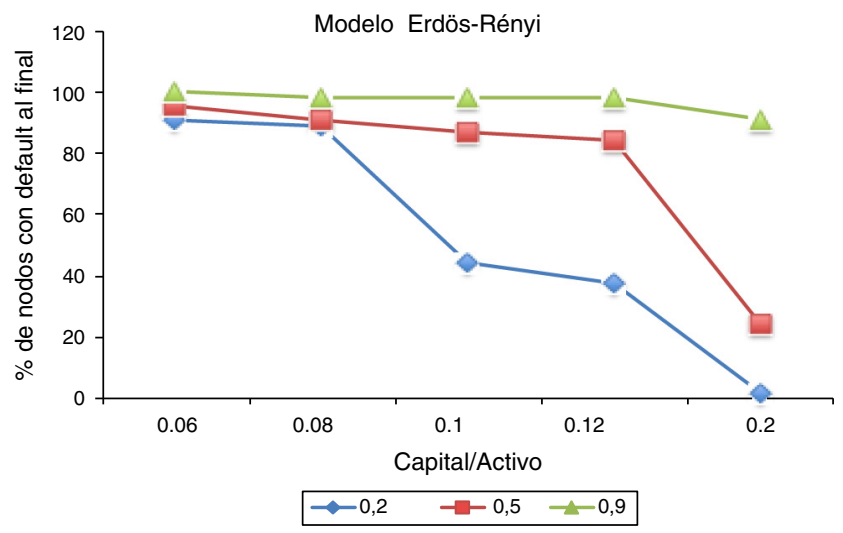

Figura 10. Número de nodos en default vs severidad de choque idiosincrático. Modelo Erdös-Rényi. Fuente: elaboración propia con resultado de simulador.

Con la finalidad de obtener mayor consistencia se consideran los valores promedio de la figura 10, donde se plantea la relación de nodos que al final del proceso estarán contagiados contra la proporción capital/activo. Se puede observar como a medida que aumenta el número promedio de los bancos con choque inicial (0.2, 0.5, 0.9), el número de nodos contagiados al término del proceso también aumenta. Además, a medida que el cociente capital/activo (en cada caso de nodos infectados inicialmente) se incrementa, el efecto de contagio sobre los otros nodos va disminuyendo.

Combinando los resultados de las dos fases anteriores se tiene que un incremento en la volatilidad o un incremento considerable de la deuda de los bancos por situaciones propias o externas provocarían la entrada en default de unos bancos al principio del proceso. Entonces, si el choque inicial de probabilidad de incumplimiento en los bancos es del $20 \%$, un incremento de la proporción capital/activos que pasa del $8 \%$ al $10 \%$ reduce la probabilidad de bancos contagiados al final 
del proceso del $80 \%$ al $40 \%$; en un caso extremo, un valor del $20 \%$ de capital/activo para el mismo prácticamente evitaría el contagio en el sistema. Sin embargo, en otra situación extrema donde el choque inicial llega al $90 \%$ de los bancos, independientemente de cuán alta sea la proporción capital/activo, no se podrá evitar el contagio a todo el sistema.

\section{Conclusiones}

Uno de los puntos básicos de los acuerdos de Basilea es el establecimiento de las metodologías para determinar el capital regulatorio de las instituciones financieras y su proporción respecto a los activos de riesgo. El presente estudio es consistente con las medidas adoptadas para el control del riesgo sistémico.

En las últimas décadas el sistema bancario mexicano ha pasado por diversas etapas que van desde la nacionalización, la reprivatización, las recurrentes crisis económico-financieras, la entrada de competencia externa con el TLCAN, hasta la apertura de diferentes nichos o poblaciones objetivo de la banca. Actualmente el sistema mexicano cuenta con 45 bancos, y a pesar de los constantes cambios, en general, la banca ha tratado de disciplinarse a las medidas propuestas por el gobierno en materia de mejorar los niveles de concentración y en materia de regulación de acuerdo a los criterios de Basilea, por lo que cada vez va resultando una banca más sólida y competitiva.

El modelo propuesto de contagio bancario consta de dos etapas. En la primera de ellas se utiliza el modelo de Merton-Credit-Monitor, que hace uso la historia de los activos y pasivos y mediante la técnica de Black-Scholes propone una metodología para determinar la probabilidad de que un banco pueden entrar en quiebra o default, en este caso por efectos externos provenientes de alta volatilidad de los activos o por el aumento considerable de sus pasivos.

En la segunda etapa se analiza el modelo de contagio financiero desde la propuesta de Dasgupta y Kaligounder, y a pesar de que el número de simulaciones no es muy grande por las limitaciones del programa ${ }^{9}$, pueden obtenerse resultados interesantes. A partir de variar distintos parámetros como la severidad del choque inicial, el tipo de choque, el porcentaje activos externos/internos, el porcentaje capital/activos se pueden extraer conclusiones de los promedios como las que se mencionan a continuación:

La probabilidad de contagiar a todo el sistema aumenta si el número de bancos que inicialmente estaban en default es alto o bien si la proporción capital/activos va disminuyendo.

Algunos casos particulares de interés son los siguientes: si el choque inicial de probabilidad de incumplimiento en los bancos es del $20 \%$ respecto del total de los bancos, entonces un incremento de la proporción capital/activos, al pasar del 8 al 10\%, reduce la probabilidad de bancos contagiados al final del proceso casi a la mitad (desde más del $80 \%$ a cerca del $40 \%$ ). Por otra parte, un incremento de hasta el $20 \%$ de dicha proporción prácticamente evitaría el contagio en el sistema. En una situación extrema donde el choque inicial de los bancos afectados sea del $90 \%$ del total de los bancos, no importa qué tan alto sea la proporción capital/activo, no se podrá evitar el contagio a todo el sistema.

En el contexto de los bancos de México podemos concluir que una proporción del $20 \%$ de capital/activos prácticamente evitaría una propagación de un contagio, independientemente de número inicial de bancos afectados(a menos que inicialmente casi todos estuvieran en quiebra). En cambio, si el número de bancos afectados por el choque inicial pasa del $20 \%$ al $50 \%$

\footnotetext{
${ }^{9}$ Cada vez que se hace una simulación, de acuerdo al software se tiene que correr con todas las características propias de cada caso, por lo que no pueda correrse miles de veces.
} 
entonces sí hay una diferencia importante para los nodos finales, que terminan contagiados (casi el doble) cuando la diferencia de la proporción capital/activos pasa del 8 al 10\%. En resumen, el resultado final de bancos contagiados cambia si hay una diferencia importante cuando cambian las proporciones capital/activos y el tamaño de nodos infectados inicialmente.

\section{Referencias}

Comisión Nacional Bancaria y de Valores (Reporte de alertas tempranas de Basilea III, circular única y estadísticas), http://www.cnbv.gob.mx/

Secretaría de Hacienda y Crédito Público. Boletines Estadísticos de Banca Múltiple, http://portafoliodeinformacion. cnbv.gob.mx/bm1/Paginas/boletines.aspx

Basel Committee on Banking Supervision, http://www.bis.org/bcbs/index.htm

Dasgupta, B. y Kaligounder, L. (2014). On global stability of financial networks. Journal of Complex Networks, 2(3), 313-354. http://dx.doi.org/10.1093/comnet/cnu004

Erdös, P. y Rényi, A. (1960). On the evolution of random graphs. Publications of the Mathematical Institute of Hungarian Academy of Sciences, 5, 17-61.

Estrada, P. y Morales, D. (2007). La estructura del mercado interbancario y del riesgo de contagio en Colombia. Documento de trabajo Banco de España,. Estabilidad Financiera No. 17.

Gai P., Kapadia S. (2010). Contagion in financial networks. Working paper no. 383. Bank of England.

Gleeson, J., Hurd, T. R., Melnik, S. y Hackett, A. (2013). Systemic Risk in Banking Networks without Montecarlo Simulation. Advances in Networks Analysis and its Applications. Berlín: Springer-Verlag.

Hernández, F. (2001). La banca en México 1990-2000. Economía Mexicana, 10(2), 363.

Klinger T., Teply P. (2014). Systemic Risk of the Global Banking System-an agent-based network model approach. Prague Economic papers 1. http://dx.doi.org/10.18267/j.pep.471

Martínez-Jaramillo S., Alexandrova Kabadjova B., Bravo Benitez B., Solorzano Margain J.P. (2012). An Empirical Study of the Mexican Banking System's Network and its Implications for Systemic Risk. Working Papers No. 2012-07, Banco de México.

Newman, M. E. J. (2002). Spread of epidemic disease on networks. Physical Review E, 66, 016128. http://dx.doi.org/ 10.1103/PhysRevE.66.016128

Newman, M. E. J. (2003). Random graphs as models of networks. En S. Bornholdt y H. G. Schuster (Eds.), Handbook of Graphs and Networks (pp. 35-68). Berlín: Wiley-VCH.

Nier, E., Yang, J., Yorulmazer, T. y Alentorn, A. (2007). Network models and financial stability. Journal of Economics Dynamics \& Control, 31, 2033-2060. http://dx.doi.org/10.1016/j.jedc.2007.01.014

Sierra, G. y García, K. (2013). Determinación de la probabilidad de incumplimiento para empresas que cotizan en la BMV utilizando el modelo de Merton con activos con características de persistencia. Avances recientes en valuación de activos y administración de riesgos, 4, 143-176.

Solorza, M. L. (2008). Nueva banca en México: incorporación de sectores populares al financiamiento. Economía Informa, $355,108-120$.

Watts, D. J. y Strogatz, S. H. (1998). Collective dynamics of small world networks. Nature, 393, 440-442. http://dx.doi. org/10.1038/30918 